\title{
Magnetic properties of FeCo films prepared by co-sputtering and hydrogenous gas reactive sputtering
}

\author{
Xiaoxi Liu ${ }^{1}$, Akimitsu Morisako ${ }^{2}$ \\ ${ }^{1}$ Department of Information Engineering, Shinshu University, Wakasato 4-17-1, Nagano, 380-8553, Japan \\ ${ }^{2}$ Spin Device and Technology Centre, Shinshu University, Wakasato 4-17-1, Nagano, 380-8553,
}

\begin{abstract}
In this experiment, we have added $\mathrm{C}$ into FeCo films by two kind of sputtering process, the first is the conventional process of cosputtering, the second is the introducing hydrogenous gas, (acetylene gas in this experiment). The crystallographic, microstructure , and magnetic properties of the films have been compared. Although nano-crystalline films, which have much lower coercivity according to the random anisotropy theory proposed by Herzer [7], can be prepared by both processes, magnetic properties of the films prepared by the two processes are different. Magnetic soft films are successfully prepared with coercivity about 1 Oe, saturation magnetization about $23.5 \mathrm{KG}$, and real part permeability as large as 1200-1500 in the frequency range up to $2.6 \mathrm{GHz}$. X-ray photoelectron spectroscopy results show films deposited without hydrogenous gas have oxygen contamination as high as 3 at \%. Oxygen free films are found to be prepared with the introducing of acetylene gas during the sputtering process.
\end{abstract}

Index Terms — high magnetic induction films, soft magnetic properties, FeCo, magnetic write head.

\section{INTRODUCTION}

$\mathrm{S}$ OFT MAGNETIC thin films with high saturation magnetization are required for magnetic head materials in order to saturate the highly coercive media. The $\mathrm{Fe}_{1-\mathrm{x}} \mathrm{Co}_{\mathrm{x}}$ $(0.3<\mathrm{x}<0.4)$ alloy has the highest saturation flux density of $4 \pi M_{s} \geq 24 \mathrm{kG}$ in nature [1]. Much effort has been taken to introduce soft magnetic properties in FeCo thin films [2]-[4]. Additives have been considered to be an effective method because suitable additives can reduce the grain size or control the resistivity [5]. So far, additives that induce soft magnetic properties are AlO, N,etc [5],[6]. However, not all the additives can induce soft magnetic properties in $\mathrm{FeCo}$ thin films.

In this experiment, additive of $\mathrm{C}$ was introduced into the films by two processes; the first is the convenient process of co-sputtering. The second is introducing hydrogenous gas, such as acetylene into the chamber during sputtering. The magnetic properties of the films prepared by the two processes were compared. It is found that films with $4 \pi M_{s}$ of $23.5 \mathrm{kG}$ and coercivities as small as 1 Oe can be prepared by hydrogenous gas reactive sputtering. While films prepared by co-sputtering have coercivities larger than $40 \mathrm{Oe}$.

\section{EXPERIMENTAL PROCEDURE}

Thin films were prepared by a facing target sputtering system [8]. C was introduced into the films by co-sputtering and reactive gas sputtering, respectively. In the case of cosputtering, carbon chips were placed onto the FeCo targets, with the composition of $\mathrm{Fe}_{65} \mathrm{Co}_{35}$. The amount of the additive is controlled by the number of the carbon chips. In the case of gas reactive sputtering, acetylene gas, incorporated with argon gas, was introduced into the chamber. The amount of carbon addition was controlled by the acetylene gas partial pressure for the latter case. For both case, the base pressure of the system is better than $3 \times 10^{-7}$ Torr. The substrate is slide glass with the size of $35 \times 20 \mathrm{~mm}$. The thickness of the films are around $200 \mathrm{~nm}$. The static magnetic properties of the samples are evaluated by a vibrating sample magnetometer (VSM). Microstructures of the samples are observed by a transmission electron microscopy (TEM). The crystallographic properties of the films are detected by a X-ray diffractometer (XRD). The compositions of the films are measured by an X-ray photoelectron spectroscopy (XPS).

\section{RESULTS AND DISCUSSION}

Fig. 1 shows XRD diagrams of films prepared by acetylene gas reactive sputtering (a) and co-sputtering (b). For the films prepared by the two different processes, The common phenomena is that the intensity of diffraction lines decrease with the increase of the acetylene gas partial pressure or increase of the $\mathrm{C}$ composition. The diffraction lines also shifted to the lower angle direction with the increase of carbon composition in both cases. This indicates that the $b c c$ lattice expanded with introducing of acetylene gas or carbon atoms by co-sputtering. A reasonable explanation is the diffusion of carbon atoms into the $\mathrm{FeCo}$ lattice for both cases. Films prepared with the introducing of the acetylene gas less than $1 \%$ show (200) as the main diffraction line, while films prepared by co-sputtering show (110) as the main diffraction line. The detailed mechanism for the different preferential orientation introduced by the two different processes is still unclear. However, the in-plane magnetocrystalline anisotropy energy and saturation magnetostriction for cubic films with preferential orientation are depended on the preferential orientation. Hence the magnetic properties of the films, which will be discussed later, depended on the crystallographic texture [7].

It is found by TEM observations that the grain size of the films decrease with the increase of the carbon composition. Fig. 2 shows the cross-sectional TEM images of FeCo films 
without $\mathrm{C}$ addition (a), with the introducing of acetylene gas at the partial pressure of $1 \%$ (b), and with the introducing of acetylene gas at the partial pressure of $5 \%$ (c). Films without $\mathrm{C}$ addition show grains as large as $100 \mathrm{~nm}$. The grain size of the films decreases with the increase of the carbon addition. Films deposited by introducing of acetylene gas at the partial pressure of $1 \%$ show a typical nano-crystalline structure with grains in the size as small as several nanometer. With further increase of the carbon addition, the films became amorphous and no distinct grain structure is found in the films, as shown in Fig.2 (c). According to the random anisotropy theory proposed by Henzer, the magnetic softness of the films with nano-crystalline structure can be strongly enhanced even for materials have relatively lager magnetostriction like $\mathrm{FeCo}$. Although the images were not shown here, the TEM images of the films prepared co-sputtering have almost the same tendency, that is, with the increase of the carbon addition, the films first show nano-crystalline structure. Amorphous structure was observed with further increase of the carbon composition.

Fig. 3 shows the dependence of magnetization on the acetylene gas partial pressure (a) and the dependence of magnetization on the carbon composition prepared by cosputtering (b). The saturation magnetization first decreases slightly with the introducing of the acetylene gas then decrease dramatically with further introducing of the acetylene gas about $1.5 \%$. It is shown that saturation magnetization decreasing almost linearly with the increasing of the carbon composition for the films deposited by co-sputtering. According the the XPS results, the carbon composition of the films prepared by reactive gas sputtering does not linearly depended on the acetylene gas pressure. Dramatic increase of the composition of carbon is found for the acetylene gas pressure higher than $1.5 \%$. This may explain that the magnetization of films prepared by gas reactive sputtering first increase slightly then decrease dramatically with the increase of acetylene gas partial pressure, as shown in Fig.3(a).

All the films show in-plane anisotropic magnetic properties with the magnetic easy axis along the stray field direction. The stray field is due to the magnet behind the target. Fig. 4 shows the dependence of coercivity on the acetylene partial pressure (a) and the dependence of carbon composition for films prepared by co-sputtering (b). It is found that coercivity decrease with the increase of carbon addition for both films. However, films prepared by gas reactive sputtering show coercivity minimum of about 1 Oe in both easy and hard axis directions. The same films show nano-crystaline structure as shown in Fig. 2 (c). While films prepared by co-sputtering show coercivity larger than 40 Oe in both easy and hard axis directions. The decrease of the coercivity with the increase of the acetylene gas pressure, as shown in Fig.4 (a), is due to the the nan-crystalline structure, as shown in Fig.2 (b).

According to the X-ray photoelectron spectroscopy results, oxygen contamination is not only found in the films without carbon addition but also found in the films prepared by cosputtering. Films without carbon addition show the oxygen contamination as large as 3 at\%. For films deposited by cosputtering, it is found that the oxygen contamination slightly decrease from 3 at $\%$ to 2 at $\%$ with the increase of the carbon composition from 0 at $\%$ to 30 at $\%$. However, no oxygen contamination have been found for the films deposited by introducing of acetylene gas. Fig. 5 shows typical XPS spectrum from $525 \mathrm{eV}$ to $540 \mathrm{eV}$ of the films prepared by acetylene gas reactive sputtering at the acetylene gas partial pressure of $0.5 \%$ (a) and co-sputtering with the carbon composition of 7 at\% (b). According to the XPS results, it is not difficult to understand that the magnetization dependence on the acetylene gas partial pressure shown in Fig. 3 (a). Because of the oxygen contamination, films deposited without acetylene gas show relative lower saturation magnetization. It should also be noted that we have found oxygen contamination for FeCo films deposited by different systems. It seems that the base pressure of $3 \times 10^{-7}$ Torr is still not enough to eliminate the oxygen contamination in all the sputtering systems we have tried.

Considering the oxygen contamination, it may also easy to understand the dependence of coercivity on the carbon addition as shown in Fig. 4. Films free of oxygen contamination prepared by introducing of acetylene gas show relatively smaller coercivity, while films with the oxygen contamination, although those films also have nano-crystalline structure by TEM, show relatively large coercivity.

Typical hysteresis loops of the films free of oxygen contamination, (prepared with the introducing of acetylene gas at the partial pressure of $1 \%$ ), are shown in Fig. 6, inset. Coercivities of the films are 0.9 Oe and $1 \mathrm{Oe}$, in the hard and easy axis, respectively. The in-plane uniaxial anisotropy field, $H_{k}$, is about 60 Oe. The saturation magnetization of the sample is about $23.5 \mathrm{KG}$. For the same sample, we measured the hard axis magnetic permeability, both real and imaginary parts, using a permeance meter with a frequency range up to $3 \mathrm{GHz}$. The results are shown in Fig. 6.The real permeability is 12001500 ate frequency up to $2.6 \mathrm{G} \mathrm{Hz}$. We could not observe the peak of the imaginary permeability. This is reasonable because of the calculated ferromagnetic resonance frequency, $f_{F M R}=\gamma \sqrt{4 \pi M_{s} \times H_{K}}=3.4 \mathrm{GHz}$, is larger than the measurement range of the permeance meter.

\section{CONCLUSION}

In conclusion, we have compared the magnetic properties of $\mathrm{C}$ doped $\mathrm{FeCo}$ films prepared by hydrogenous gas reactive sputtering and co-sputtering. The gain sizes of the films decrease with the increase of $\mathrm{C}$ doping for both process. Films prepared by acetylene gas reactive sputtering show lower coercivity. Films with $4 \pi M_{s}$ of $23.5 \mathrm{kG}$ and coercivities as small as 1 Oe can be prepared by acetylene gas reactive sputtering, however, the samples prepared by co-sputtering have coercivities about $40 \mathrm{Oe}$ at the same saturation magnetization. XPS results show that oxygen free FeCo films can be prepared by reactive sputtering of hydrogenous gas. While films deposited by co-sputtering have impurities of oxygen as large as 3 at $\%$. 
The permeability spectra of those oxygen free films confirm that those films are promising for use in high density recording head or high frequency integrated inductors.

\section{ACKNOWLEDGMENT}

This work is supported by MEXT, grand-in-aid for Young Scientist.

\section{REFERENCES}

[1] R. M. Bozorth, Ferromagnetism, IEEE, New York, 1993.

[2] S. X. Wang, N. X. Sun, M. Yamaguchi, and S. Yabukami, "Properties of a new soft magnetic material", Nature Vol. 407, pp.150-151, 2000.

[3] G. Pan and H. Du, "( $\mathrm{FeCo} / \mathrm{Co}-\mathrm{M})_{\mathrm{n}}$ soft magnetic multilayers with uniaxial anisotropy and very high saturation magnetization," J. Appl. Phys. Vol. 93, pp.5498-5502, 2003.

[4] K. Shintaku, K. Yamakawa and K. Ouchi, "High- $B_{\mathrm{s}}$ Fe-Co-Al-O soft magnetic films," J. Appl. Phys. Vol. 93, pp. 6474-6476, 2003.

[5] S. Ikeda, I. Tagawa, Y. Uehara, T. Kubomiya, J. Kane, M. Kakehi, and A. Chikazawa, "Write head with pole tip consisting of high-Bs FeCoAlO films," IEEE Trans. Magn. Vol. 38, pp. 2219-2221, 2002.

[6] N. X. Sun and S. X. Wang, "Soft high saturation magnetization FeCoN thin films for inductive write heads," IEEE Teans. Magn. Vol. 36, pp. 2506-2508, 2000.

[7] G. Herzer, "Grain size dependence of coercivity and permeability in nanocrystalline ferromagnets," IEEE Teans. Magn. Vol. 26, pp. 1397$1402,1990$.

[8] M. Naoe, S. Yamanaka, and Y. Hoshi, "Facing targets type of sputtering method for deposition magnetic metal films at low temperature and high rate," IEEE trans. Magn. , Vol. MAG-16, No. 5, Pp. 646-648, Sep. 1980.

Manuscript received March 3, 2008 (date on which paper was submitted for review). Corresponding author: X. L. Author (e-mail: liu@cs.shinshuu.ac.jp; optional phone: +81-26-269-5480; optional fax: +81-26-269-5495).

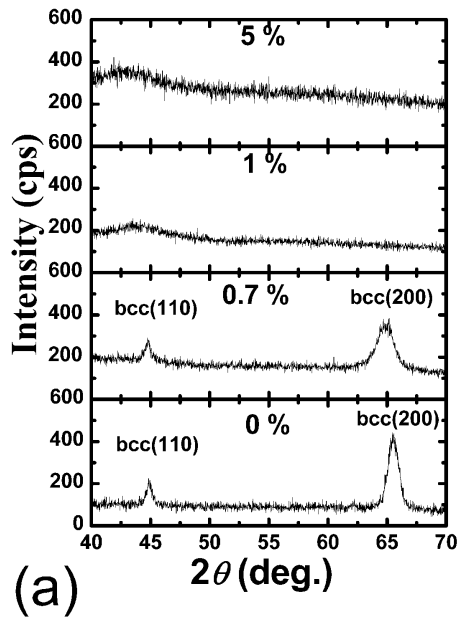

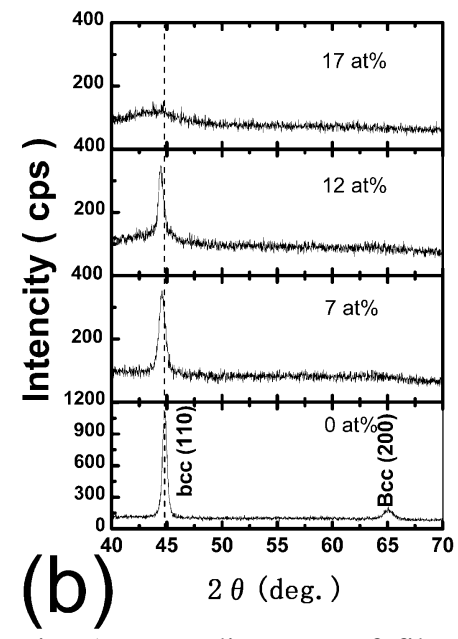

Fig. 1 XRD diagrams of films prepared by acetylene gas reactive sputtering (a) and co-sputtering (b).
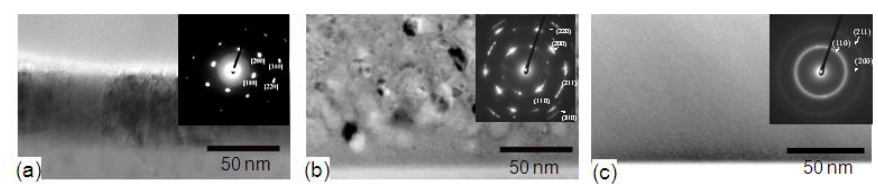

Fig. 2 Cross-sectional TEM images of FeCo films without $\mathrm{C}$ addition (a), with introducing of acetylene gas at the partial pressure of $1 \%$ (b), and the introducing of acetylene gas at the partial pressure of $5 \%$ (c).
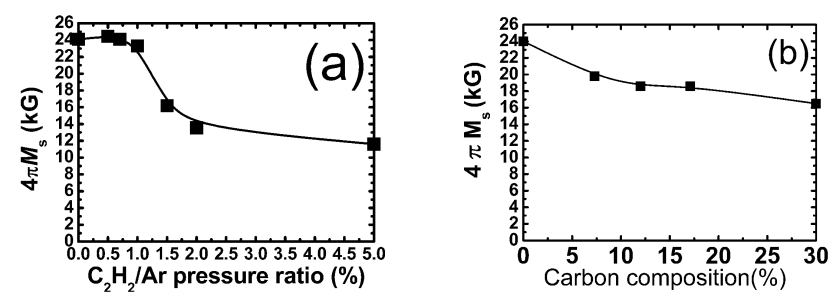

Fig. 3 the dependence of magnetization on the acetylene gas partial pressure (a) and the dependence of magnetization on the carbon composition prepared by co-sputtering (b).
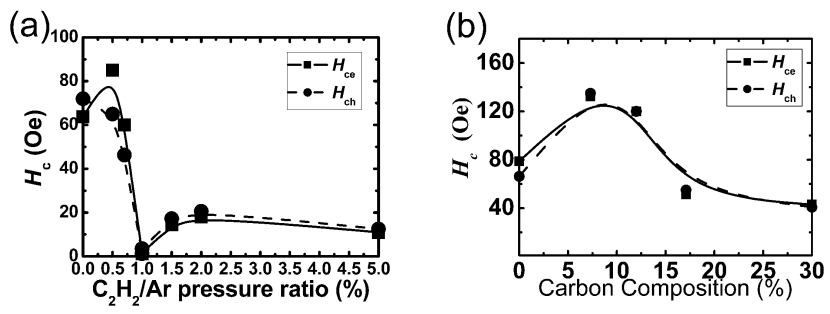

Fig. 4 the dependence of coercivity on the acetylene partial pressure (a) and the dependence of carbon composition for films prepared by co-sputtering (b). 


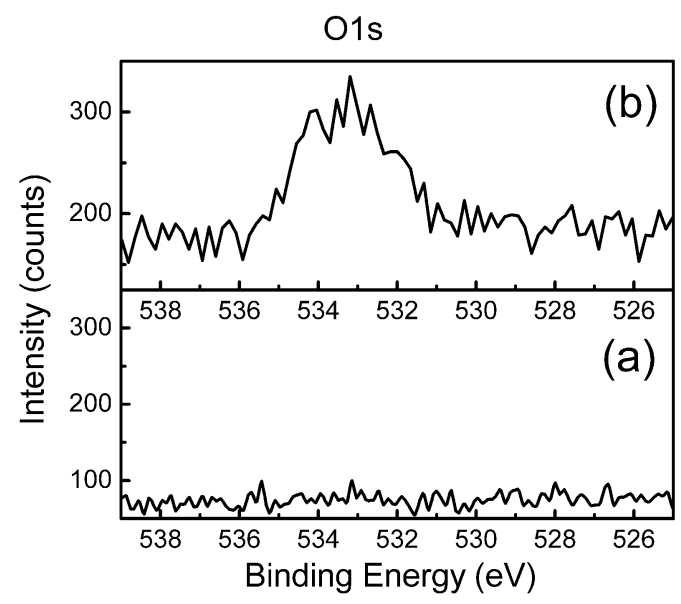

Fig. 5 typical XPS spectrum from $525 \mathrm{eV}$ to $540 \mathrm{eV}$ of the films prepared by acetylene gas reactive sputtering at the acetylene gas partial pressure of $0.5 \%$ (a) and co-sputtering with the carbon composition of 7 at\% (b).

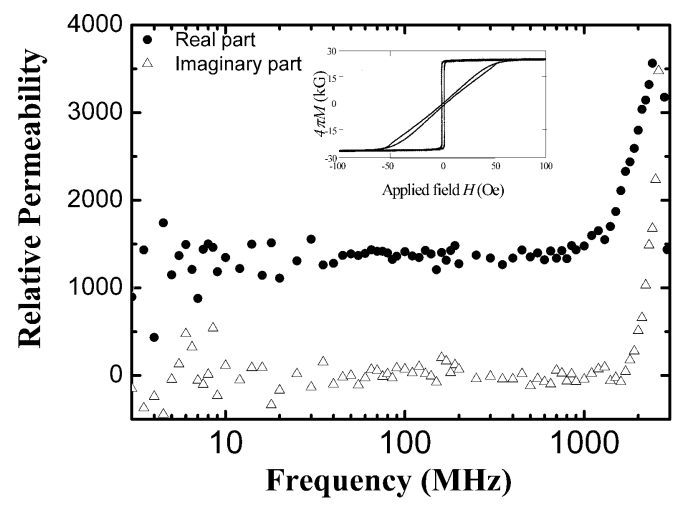

Fig. 6 Relative permeability versus frequency for films prepared by introducing of acetylene gas at the partial pressure of $1 \%$. Inset, $\mathrm{M}-\mathrm{H}$ loops of the films. 